Classification

Physics Abstracts

$05.50-02.50$

\title{
Replica field theory for random manifolds
}

\author{
Marc Mézard ( $\left.{ }^{1}\right)$ and Giorgio Parisi $\left({ }^{2}\right)$ \\ (1) Laboratoire de Physique Théorique de l'Ecole Normale Supérieure (*), 24 rue Lhomond, \\ 75231 Paris Cedex 05, France \\ (2) Dipartimento di Fisica, Università di Roma II, Via E. Carnevale, Roma 00173, Italy
}

(Received 14 January 1991, accepted 28 February 1991)

\begin{abstract}
We consider the field theory formulation for manifolds in random media using the replica method. We use a variational (Hartree-Fock like) method which shows that replica symmetry is spontaneously broken. A hierarchical breaking of symmetry allows one to take into account the existence of many metastable states for the manifold, and to recover the results of the Flory scaling arguments for the wandering exponent. This field theoretic derivation of Flory results opens the way to computing corrections to these exponents.
\end{abstract}

\section{Introduction.}

A crucial open problem in statistical physics consists in finding the behaviour of fluctuating manifolds which are pinned by quenched random impurities. For example the case of unidimensional manifolds corresponds to directed polymers in a random potential [1], a problem which is also related to surface growth [2], randomly stirred fluids described by Burgers' equation [3, 4], and spin glasses [5]. In the other extreme case where the manifold's dimension $D$ is one less than the total dimension of the space $d$, the manifold can be seen as an interface separating for instance two phases of a ferromagnet.

Actually all these problems can be studied within the same framework (for recent reviews see [6]). Moreover the fascinating problem of self interacting random manifolds (in particular of self interacting heteropolymers [7]) is also deeply related to these problems. Unfortunately in spite of the serious efforts which have been devoted to various kinds of manifolds in random potentials, the situation is still confused and conflicting results have been obtained. As an example we can concentrate on the wandering exponent $\zeta$ which characterizes the transverse fluctuations of the manifold at large distances, in the case where the manifold is an interface of the random field Ising model (RFIM). Simple scaling arguments suggest a value for $\zeta$ often called the Flory result [8], which is in this case : $\zeta^{\mathrm{F}}=(5-d) / 3$. On the other hand most of the attempts so far to derive this exponent from a microscopic theory using field theoretic methods [9-11] disagree with this result and give $\zeta=(5-d) / 2$. This value of

(*) Unité propre du Centre National de la Recherche Scientifique, associée à l'Ecole Normale Supérieure et à l'Université de Paris Sud. 
$\zeta$ can be obtained using the supersymmetric approach to the random field Ising model, under the crucial (and wrong) hypothesis [12] of the existence of only one solution to the mean field equations.

In this paper we reconsider the replica field theory for the general problem of fluctuating manifolds in a quenched random potential. We have suggested recently that a crucial ingredient, replica symmetry breaking (RSB) [13], was lacking in previous field theoretical analysis. Physically it is natural to suppose that a manifold in presence of quenched disorder can have many equilibrium points [14]. If this happens, standard perturbation theory is unable to take into account the fluctuations between free energy minima which are far from each other in phase space. The most adequate formalism to handle this kind of problems is the replica method with broken replica symmetry [15]. The aim of this paper is to extend our recent results [16] and to give a physical interpretation for the breaking of replica symmetry.

Technically, the problem is studied with a variational Hartree-Fock like method of the same kind of the one used by Shakhnovich and Gutin [7] in their study of heteropolymer folding. Using a hierarchical breaking of replica symmetry familiar from spin glass theory, we find back the Flory result for the exponent $\zeta$ in a simple way. Moreover this variational method is shown to be exact when the codimension of the manifold $(N=d-D)$ goes to infinity. In principle the stability of the RSB solution can be studied and this should open the way to a systematic evaluation of the corrections to $\zeta$. This is a complicated computation which can be done using techniques similar to those of [17] and it is left for future study.

The paper is organized as follows. In section 2 we define the systems we want to study and the problems; we briefly state the scaling arguments. In section 3 we introduce the field theory representation of the replicated system; we describe the variational approach and show that it becomes exact in the large $N$ limit. Section 4 describes the replica symmetric solution and its stability. In section 5 we discuss the solution with replica symmetry breaking. The physical interpretation of this breaking is analyzed in section 6 . Section 7 contains a discussion and some prospects for future developments. Some technical points are discussed in three appendices dedicated respectively to the fluctuations around the saddle point, to the evaluation of the inverse of a hierarchical matrix and to the computation of the probability distribution of the field.

\section{The model : definitions and scaling argument.}

We consider a $D$ dimensional manifold in a $N+D=d$ dimensional space, in the solid on solid approximation: the manifold is represented by a $N$ component vector field $\omega(x)$ (the subspace spanned by $\omega$ will be called the transverse space), where $x$ is the $D$ dimensional vector of internal coordinates (called the longitudinal directions). The Hamiltonian describing the system is the sum of a rigidity term and an external potential. In the continuum limit it is written as :

$$
h[\boldsymbol{\omega}]=\frac{1}{2} \int \mathrm{d} x \sum_{\mu=1}^{D}\left(\frac{\partial \boldsymbol{\omega}}{\partial x_{\mu}}\right)^{2}+\int \mathrm{d} x V(x, \boldsymbol{\omega}(x)) .
$$

Whenever this will be needed, we shall assume that this Hamiltonian is regularized at short distances through the introduction of a lattice on scales $|x| \sim a$. For the sake of regularizing intermediate steps of the computation, we shall also add a small mass term, i.e. consider the Hamiltonian :

$$
H[\omega]=h[\omega]+\frac{\mu}{2} \int \mathrm{d} x \omega(x)^{2},
$$

but eventually we shall be interested in the $\mu \rightarrow 0$ theory. 
The potential $V(x, \omega)$ is a quenched random variable with a Gaussian distribution of mean zero. In this paper we consider only the cases where the correlations of the potential are :

$$
\left.\overline{V(x, \boldsymbol{\omega}) V\left(x^{\prime}, \boldsymbol{\omega}^{\prime}\right.}\right)=-\delta^{(D)}\left(x-x^{\prime}\right) N f\left(\frac{\left[\boldsymbol{\omega}-\boldsymbol{\omega}^{\prime}\right]^{2}}{N}\right) .
$$

(The effect of having a non Gaussian distribution for $V$ with a long tail are discussed in [18]).

The $N$ dependence in equation (2.3) has been chosen such that the large $N$ limit of the theory will be well defined. In most of the cases the relevant part of the correlation function of the potential is its asymptotic behaviour for large transverse distances, which we suppose to be described by a power law:

$$
f\left(\frac{\omega^{2}}{N}\right) \stackrel{\omega^{2} \gg 1}{\rightarrow} \frac{g}{2(1-\gamma)}\left(\frac{\omega^{2}}{N}\right)^{(1-\gamma)},
$$

where $g$ is a positive coupling constant (a negative value of $g$ corresponds to purely immaginary noise, which is a quite different problem [19]). For $\gamma>1$ we shall need to remember that $f$ is regular at small arguments (see Fig. 1). The case $\gamma=0$ is soluble and the results agree with the explicit computation of [20].

The general model is mainly described by three parameters : $D$ is the internal dimension of the manifold, $N=d-D$ is its codimension, and $\gamma$ characterizes the large distance correlations of the potential. Special cases of particular interest are :

$-N=1$ : the manifold is an interface. For the RFIM the potential $V(x, \omega)$ is basically proportional to the integral of the random field from $\omega=\infty$ to $\omega=\omega(x)$, minus the integral from $\omega=-\infty$ to $\omega=\omega(x)$. This leads to $\gamma=1 / 2$.

$-D=1:$ this is the case of the directed polymer. The most studied such problem is when the disorder is local i.e. :

$$
\left.\overline{V(x, \boldsymbol{\omega}) V\left(x^{\prime}, \boldsymbol{\omega}^{\prime}\right.}\right)=c^{l} \delta\left(x-x^{\prime}\right) \delta\left(\boldsymbol{\omega}-\boldsymbol{\omega}^{\prime}\right) .
$$

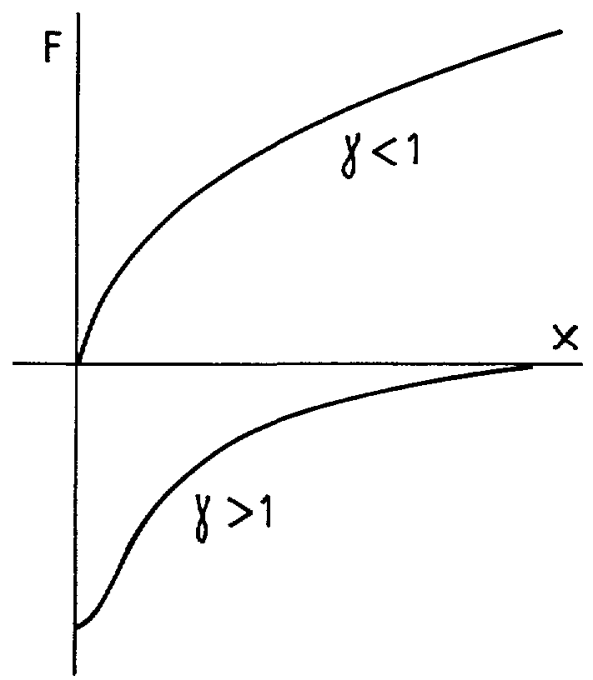

Fig. 1. - Schematic behaviour of the correlation of the disorder $f(x)$, defined in (2.3) in the cases where $\gamma<1$ and $\gamma>1$. In the latter case the asymptotic behaviour $\frac{g}{2(1-\gamma)} x^{1-\gamma}$ is regularized at short transverse distances (see (5.22)). 
From dimensional analysis one expects this case to be characterized by $\gamma=1+N / 2$. This characterization is correct within the variational scheme we shall use hereafter.

The basic questions one asks about such systems concern both their thermodynamics and the transverse fluctuations of the manifold at large distance. The partition function can be defined in the usual way: we consider the system in a longitudinal box of size $L^{D}$ (the field $\omega$ vanishes whenever $\left|z^{\mu}\right|=L / 2$ ), and :

$$
Z=\int d[\omega] \mathrm{e}^{-\beta H[\omega]}
$$

The transverse fluctuations at large distances are characterised by the exponent $\zeta$ :

$$
C(\ell, T, a) \equiv \overline{\left\langle[\omega(x+\ell)-\boldsymbol{\omega}(x)]^{2}\right\rangle} \stackrel{\ell}{\langle a} \ell^{2 \zeta}
$$

(Throughout this paper we denote the thermal averages by \langle\rangle and the averages over various realizations of the quenched potential by $(\overline{)})$.

Another exponent of interest characterizes the sample to sample fluctuations of the free energy $F=-T \log Z$ :

$$
\overline{F(L)}^{2}-\overline{F(L)}^{2} \sim L^{2 \chi}
$$

The critical exponent $\chi$ can be related to $\zeta$ by a simple scaling formula :

$$
\chi=2 \zeta+D-2 .
$$

There is a general agreement on the fact that, if $D>4$ the exponent $\zeta$ is zero (at least for $g$ not too large).

For completeness let us briefly recall a scaling argument which leads to the Flory conjecture for the value of the exponent $\zeta$ for $D<4$. To compute the transverse fluctuations $C(\ell, T, a)$ defined in (2.7), in the large $\ell$ limit, we rescale $x, \omega$ and $V$ to:

$$
\begin{aligned}
x & =\ell x^{\prime} \\
\boldsymbol{\omega} & =\ell^{\zeta} \boldsymbol{\omega}^{\prime}, \\
V(x, \boldsymbol{\omega}) & =\ell^{\lambda} V^{\prime}\left(x^{\prime}, \boldsymbol{\omega}^{\prime}\right) .
\end{aligned}
$$

The exponent $\lambda$ is chosen in such a way that $V^{\prime}$ has the same distribution as the original $V$, which imposes:

$$
\lambda=-\frac{D}{2}+\zeta(1-\gamma)
$$

For the following choice of the exponent $\zeta$ :

$$
\zeta=\zeta^{\mathrm{F}} \equiv \frac{4-D}{2(1+\gamma)},
$$

the rescaling can be absorbed into a temperature rescaling so that:

$$
C(\ell, T, a)=C\left(1, \frac{T}{\ell^{\mathrm{F}}}, \frac{a}{\ell}\right),
$$

where $\chi^{\mathrm{F}}$ is related to $\zeta^{\mathrm{F}}$ through the general formula (2.9), giving:

$$
\chi^{F}=\frac{2+\gamma(D-2)}{1+\gamma}
$$


This argument would prove that the Flory value for $\zeta$ is exact if the zero temperature continuum limit of the theory were well defined, which is far from obvious in general.

A relatively well understood counterexample is the case of random directed polymer in $1+1$ dimensions $(D=N=1)$, with local disorder $(\gamma=3 / 2)$. One finds $\zeta^{\mathrm{F}}=3 / 5$ while the exact result is known to be $\zeta=2 / 3[4,21]$. Actually it can be shown that the continuum limit of the theory is ill defined at zero temperature, using Kardar's Bethe Ansatz approach [21].

\section{Replicas and the variational approach.}

3.1 THE REPLICA APPROACH. - In order to get the typical properties of the manifold we use the replica method. We introduce $n$ copies of the system and compute the resulting partition function :

$$
\overline{Z^{n}}=\int d\left[\omega_{1}\right] \cdot d\left[\omega_{n}\right] \mathrm{e}^{-\beta H_{n}},
$$

where the Hamiltonian in replica space is :

$$
\begin{aligned}
H_{n}=\frac{1}{2} \int \mathrm{d} x \sum_{a=1}^{n} \sum_{\mu=1}^{D}\left(\frac{\partial \boldsymbol{\omega}_{a}}{\partial x_{\mu}}\right)^{2}+\frac{\mu}{2} \int \mathrm{d} x \sum_{a=1}^{n}\left(\boldsymbol{\omega}_{a}(x)\right)^{2}+ \\
\quad+\frac{\beta}{2} \int \mathrm{d} x \sum_{a, b} N f\left(\frac{\left[\boldsymbol{\omega}_{a}(x)-\boldsymbol{\omega}_{b}(x)\right]^{2}}{N}\right) .
\end{aligned}
$$

The average over the random potential leads to an attraction between all pairs of replicas.

The usual perturbative treatment of the Hamiltonian $H_{n}$ consists in expanding $f$ in powers of $\omega$; the quadratic part gives the free propagator $F_{a b}$.

$$
F_{a b}(k)=\frac{\delta_{a b}}{k^{2}+n 2 \beta f^{\prime}(0)}+\frac{2 \beta f^{\prime}(0)}{k^{2}\left(k^{2}+n 2 \beta f^{\prime}(0)\right)}
$$

For $n \rightarrow 0$, the presence of the $1 / k^{4}$ term immediately leads to $\zeta=(4-D) / 2$. This result has been shown to hold to all orders of perturbation theory [11], provided that the propagator has a replica symmetric structure $\left(F_{a b}(k)=F_{1}(k) \delta_{a b}+F_{2}(k)\right)$.

This result is clearly surprising, especially because the large distance correlations of the potential (i.e. the behaviour of $f$ at large argument) does never appear. Also the effects of the existence of several metastable states cannot be handled by such a perturbative expansion.

3.2 VARIATIONAL METHOD. - In order to go beyond perturbation theory, we shall now use a variational method for the replicated Hamiltonian. As a trial Hamiltonian we take :

$$
h_{\sigma}=\frac{1}{2} \int \mathrm{d} x \sum_{a=1}^{n} \sum_{\mu=1}^{D}\left(\frac{\partial \boldsymbol{\omega}_{a}}{\partial x_{\mu}}\right)^{2}+\frac{\mu}{2} \int \mathrm{d} x \sum_{a=1}^{n}\left(\boldsymbol{\omega}_{a}(x)\right)^{2}-\frac{1}{2} \int \mathrm{d} x \sum_{a, b} \sigma_{a b} \boldsymbol{\omega}_{a}(x) \cdot \boldsymbol{\omega}_{b}(x),
$$

where $h_{\sigma}$ depends on a set of $n(n+1) / 2$ variational parameters which build the symmetric matrix $\sigma_{a b}$. It can be shown that the most general quadratic Hamiltonian reduces to the above form at stationarity.

The variational free energy is :

$$
F=\left\langle H_{n}-h_{\sigma}\right\rangle_{h_{\sigma}}-\frac{1}{\beta} \log \left(\int d\left[\omega_{1}\right] . d\left[\omega_{n}\right] \mathrm{e}^{-\beta h_{\sigma}}\right),
$$


where the expectation value \langle\rangle$_{h_{\sigma}}$ is taken with respect to a measure $\exp \left(-\beta h_{\sigma}\right)$. To compute $F$ it is useful to introduce the propagator of $h_{\sigma}$.

$$
G_{a b}(k)=\left(\left[\left(k^{2}+\mu\right) I-\sigma\right]^{-1}\right)_{a b} .
$$

In order to compute the expectation value $\left\langle H_{n}-h_{\sigma}\right\rangle_{k_{\sigma}}$ in (3.5), we expand $f$ in series, compute the expectation value of each term and resum the series. The final result for the free energy density is :

$$
\begin{aligned}
\frac{F}{N L^{d}}=c^{\mathrm{t}}+\frac{1}{2 \beta} \int \mathrm{d} k\left(k^{2}+\mu\right) & \sum_{a} G_{a a}(k)-\frac{1}{2 \beta} \int \mathrm{d} k \operatorname{Tr} \log G(k)+ \\
& +\frac{\beta}{2} \sum_{a, b} \hat{f}\left(\frac{1}{\beta} \int \mathrm{d} k\left[G_{a a}(k)+G_{b b}(k)-2 G_{a b}(k)\right]\right),
\end{aligned}
$$

where the integration measure $\mathrm{d} k$ stands for $\mathrm{d} k^{\mathrm{l}} \cdot \mathrm{d} k^{D} /(2 \pi)^{D}$, and :

$$
\hat{f}(x)=\frac{1}{\Gamma(N / 2)} \int_{0}^{\infty} \mathrm{d} \alpha \alpha^{\frac{N}{2}-1} \mathrm{e}^{-\alpha} f\left(\frac{2 \alpha}{N} x\right) .
$$

The effective interaction $\hat{f}$ is similar to $f$. For large argument it has the same kind of power law behaviour:

$$
\hat{f}(x) \stackrel{x \gg 1}{\sim} \frac{\hat{g}}{2(1-\hat{\gamma})} x^{1-\hat{\gamma}}
$$

where :

$$
\begin{array}{ccc}
\hat{\gamma}=\gamma & \text { if } & \gamma \leqslant \frac{N}{2}+1 \\
\hat{\gamma}=\frac{N}{2}+1 & \text { if } & \gamma \geqslant \frac{N}{2}+1 .
\end{array}
$$

(For $\gamma=\frac{N}{2}+1$ there are logarithmic corrections). Hereafter we shall always work in the regime where $\hat{\gamma}=\gamma$. We shall also use the notation:

$$
\hat{g}=g \frac{\Gamma(1-\gamma+N / 2)}{\Gamma(N / 2)}\left(\frac{N}{2}\right)^{-1+\gamma}
$$

For $N$ going to infinity $\hat{f}$ becomes identical to $f$.

The stationarity condition of the free energy $F$ with respect to the parameters $\sigma_{a b}$ gives :

$$
\begin{gathered}
\sigma_{a b}=2 \beta \hat{f}^{\prime}\left(\frac{1}{\beta} \int \mathrm{d} k\left[G_{a a}(k)+G_{b b}(k)-2 G_{a b}(k)\right]\right), \quad a \neq b \\
\sigma_{a a}=-\sum_{b(\neq a)} \sigma_{a b} .
\end{gathered}
$$

The saddle point equations (3.6), (3.12) have a simple interpretation: $G$ is the variational Ansatz for the full propagator. From (3.6), $\sigma$ is the corresponding self energy. The equation for $\sigma$ just expresses the self energy as a sum of tadpole graphs (see Fig. 2) in terms of the full propagator, as can be seen by expanding this equation in powers of $G$. This is basically the Hartree-Fock approximation. 


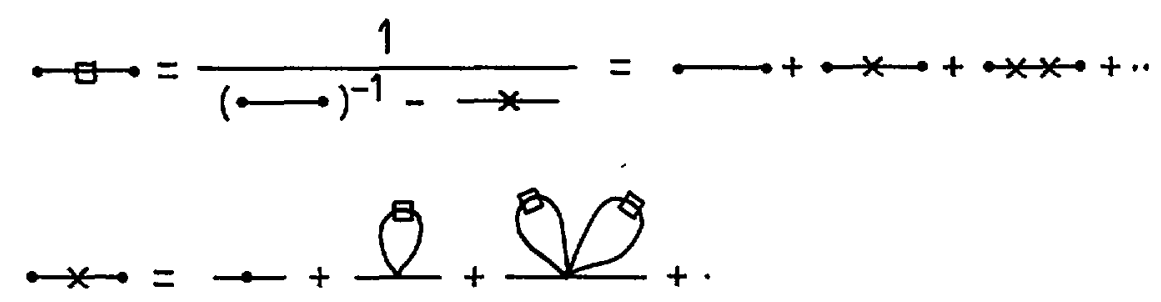

Fig. 2. - Diagrammatical interpretation of the stationarity equations $(3.12):(\rightarrow \square)$ is the full propagator, $(\longrightarrow)$ is the propagator without disorder, and $(\bullet x \bullet)$ is the self energy. Within the Gaussian approximation, the self energy is the sum of all the generalized tadpole diagrams.

3.3 LARGE $N$ LIMIT : AUXILIARY FIELDS. - It is well known in field theory that the HartreeFock approximation becomes exact when the number $N$ of components of the field is large. Therefore we expect the variational method to become exact in large dimensions. A convenient proof of this result in this limit can be obtained using another representation of the problem, i.e. introducing $n(n+1) / 2$ auxiliary fields :

$$
\tau_{a b}(x)=\frac{1}{N} \omega_{a}(x) \cdot \omega_{b}(x) \quad(a \leqslant b) .
$$

The constraints defining the field are implemented by a set of Lagrange multipliers $s_{a b}(x)$, and we get :

$$
\begin{aligned}
\overline{Z^{n}=\int} \prod_{a=1}^{n} d\left[\dot{\omega}_{a}\right] \int \prod_{a \leq b}\left(d\left[r_{a b}\right] d\left[s_{a b}\right]\right) \times & \times \\
& \times \exp \left(-\frac{\beta}{2} \sum_{a, b} \int \mathrm{d} x s_{a b}(x)\left[N r_{a b}(x)-\boldsymbol{\omega}_{a}(x) \cdot \boldsymbol{\omega}_{b}(x)\right]\right) \\
\exp \left(-\frac{\beta}{2} \int \mathrm{d} x \sum_{a=1}^{n} \sum_{\mu=1}^{D}\left(\frac{\partial \boldsymbol{\omega}_{a}}{\partial x_{\mu}}\right)^{2}-\frac{\beta \mu}{2} \int \mathrm{d} x \sum_{a=1}^{n}\left(\boldsymbol{\omega}_{a}(x)\right)^{2}-\right. & \left.-\frac{\beta^{2}}{2} \int \mathrm{d} x \sum_{a, b} N f\left(r_{a a}(x)+r_{b b}(x)-2 r_{a b}(x)\right)\right)
\end{aligned}
$$

Integrating out the fields $\omega_{a}$, we arrive at :

where:

$$
\overline{Z^{n}}=\int \prod_{a \leqslant b}\left(d\left[r_{a b}\right] d\left[s_{a b}\right]\right) \mathrm{e}^{N G[r, s]},
$$

$$
\begin{aligned}
& G[r, s]=-\frac{\beta}{2} \sum_{a, b} \int \mathrm{d} x s_{a b}(x) r_{a b}(x)-\frac{\beta^{2}}{2} \int \mathrm{d} x \sum_{a, b} f\left(r_{a a}(x)+r_{b b}(x)-2 r_{a b}(x)\right)+S[s] \\
& \mathrm{e}^{S[s]}=\int d\left[\omega_{1}\right] . . d\left[\omega_{n}\right] \times \\
& \times \exp \left(-\frac{\beta}{2} \sum_{a, b} \int \mathrm{d} x \omega_{a}(x)\left(\left(-\nabla^{2}+\mu\right) \delta_{a b}-s_{a b}(x)\right) \omega_{b}(x)\right)
\end{aligned}
$$


In the new representation (3.15) the large $N$ limit can be handled by a saddle point method. It is not surprising to find that there exists a uniform saddle point for $s$ and $r$, for which the saddle point equations reduce identically to the stationarity equations obtained before with the variational trial Hamiltonian $h_{\sigma}$, with $s_{a b}=\sigma_{a b}$. The only difference is that the function $\hat{f}$ of (3.12) is replaced by $f$, but $f$ and $\hat{f}$ become identical in the large $N$ limit. Apart from showing that the equations (3.12) are exact in large dimensions $(N \rightarrow \infty)$, the representation (3.15) is also well suited for performing $1 / N$ expansions. The general form of the quadratic fluctuations around a uniform saddle point in (3.15) is worked out in appendix $\mathbf{I}$.

\section{Replica symmetric solution.}

We now proceed to discuss the solution of the stationarity equations (3.6), (3.12) within the assumption of replica symmetry: $\sigma_{a b}=\sigma(a \neq b)$. The equations reduce to :

$$
\begin{gathered}
\sigma=2 \beta \hat{f}^{\prime}\left(\frac{1}{\beta} \int \mathrm{d} k\left[G_{a a}(k)+G_{b b}(k)-2 G_{a b}(k)\right]\right), \quad a \neq b \\
G_{a b}(k)=\left(\left[\left(k^{2}+\mu\right) I-\sigma\right]^{-1}\right)_{a b}=\frac{\delta_{a b}}{k^{2}+\mu-n \sigma}+\frac{\sigma}{\left(k^{2}+\mu\right)\left(k^{2}+\mu-n \sigma\right)},
\end{gathered}
$$

which gives in the $n$ going to zero limit :

$$
\sigma=2 \beta \hat{f}^{\prime}\left(\frac{2}{\beta} \int \mathrm{d} k \frac{1}{k^{2}+\mu}\right) .
$$

The solution is :

$$
\begin{array}{lrr}
\sigma=c^{\mathrm{t}} \hat{g} \beta^{\mathrm{l}+\gamma} \mu^{\gamma \frac{2-D}{2}(\mu \rightarrow 0)} 0 & (D<2) \\
\sigma=c^{\mathrm{t}} \hat{g} \beta^{\mathrm{t}+\gamma} a^{\gamma(D-2)} & (2<D<4),
\end{array}
$$

where $a$ is the ultraviolet cut-off. The correlation function is :

$$
\overline{\left\langle[\boldsymbol{\omega}(x)-\boldsymbol{\omega}(y)]^{2}\right\rangle}=\lim _{n \rightarrow 0} \frac{1}{n} \sum_{a} \int \mathrm{d} k 2(1-\cos [k(x-y)]) G_{a a}(k),
$$

which leads to the exponent:

$$
\begin{array}{ll}
\zeta_{\mathrm{RS}}=\frac{4-D}{2} & 4>D>2 \\
\zeta_{\mathrm{RS}}=\frac{2-D}{2} & 2>D .
\end{array}
$$

In order to test the validity of this replica symmetric solution, one possibility is to use the fact that this solution is a saddle point of (3.15) for large $N$. The Gaussian fluctuations around a generic uniform saddle point are analyzed in Appendix I.

It is shown that the modes with different $k$ decouple, so that one is left with one $n(n-1) / 2 \times n(n-1) / 2$ matrix of fluctuations for each mode, $M_{a b, c d}(k)$. For the special case of the fluctuations around a replica symmetric saddle point, the matrix elements of 
$M_{a b, c d}(k)$ may take three different values, which we denote respectively $P(k), Q(k)$, $R(k)$ depending on how many indices are common to the pairs $\{a b\}$ and $\{c d\}$ :

$$
\begin{array}{ll}
|\{a b\} \cap\{c d\}|=2 & P(k)=1-2 \beta^{2} h^{2} I(k) \\
|\{a b\} \cap\{c d\}|=1 & Q(k)=-\frac{\beta^{2}}{2} h^{2} I(k) \\
|\{a b\} \cap\{c d\}|=0 & R(k)=0,
\end{array}
$$

where :

$$
\begin{aligned}
h^{2} & =\left|f^{\prime \prime}\left(\frac{2}{\beta} \int \mathrm{d} k \frac{1}{k^{2}+\mu}\right)\right| \\
I(k) & =\int d^{D} k^{\prime} \frac{1}{k^{\prime 2}+\mu} \frac{1}{\left(k-k^{\prime}\right)^{2}+\mu}
\end{aligned}
$$

The eigenvalues of such a matrix have been computed in a different context by De Almeida and Thouless [22]. For $n \rightarrow 0$ there are two different eigenvalues:

$$
\begin{aligned}
\text { Longitudinal: } & \lambda_{1}(k)=P(k)-4 Q(k)+3 R(k)=1 \\
\text { Replicon: } & \lambda_{2}(k)=P(k)-2 Q(k)+R(k)=1-\beta^{2} h^{2} I(k) .
\end{aligned}
$$

The longitudinal modes are always stable. As for the transverse (or « replicon ») modes the most dangerous one is around $k=0$ which gives:

$$
\begin{aligned}
D<2: & \lambda_{2}(k) \stackrel{k \rightarrow 0}{\sim} 1-c^{t} \mu^{[(1+\gamma)(2-D)+(D-4)] / 2} \\
2<D<4: & \lambda_{2}(k) \stackrel{k \rightarrow 0}{\sim} 1-c^{\mathrm{t}} a^{-[(1+\gamma)(2-D)+(D-4)]}
\end{aligned}
$$

The stability depends on the sign of $(1+\gamma)(2-D)+(D-4)=-\chi^{F}(1+\gamma)$, i.e. it is determined by the sign of $\chi^{\mathrm{F}}$, where $\chi^{\mathrm{F}}$, which has been defined in $(2.14)$, is the Flory exponent for the free energy fluctuations (when it is positive). We finally find that :

- If $\chi^{\mathrm{F}}>0$, the replica symmetric saddle point is unstable at least in the continuum limit $(a \rightarrow 0)$.

- If $\chi^{\mathrm{F}}<0$, this saddle point is stable.

\section{Replica symmetry breaking solution.}

5.1 HierarCHICAL BREAKING. - Within the variational method, the self energy $\sigma_{a b}$ is a momentum independent $n \times n$ symmetric matrix, which satisfies (3.6), (3.12). We shall now look for solutions of these equations, in the limit $n \rightarrow 0$, using the hierarchical Ansatz of replica symmetry breaking which has been successful in the mean field theory of spin glasses. We shall not explain the details of this Ansatz here since there are recent reviews [13].

In the $n \rightarrow 0$ limit the matrix is parametrized by a function $\sigma(u)$ defined for $u$ in the interval $[0,1]$, and a number $\tilde{\sigma}$ which gives the diagonal elements of $\delta_{a b}$. The second equation in (3.12) gives:

$$
\tilde{\sigma}=\int_{0}^{1} \mathrm{~d} u \sigma(u)
$$

(the replica symmetric Ansatz corresponds to the special case of a constant $\sigma(u)$ function).

In order to get the propagator $G_{a b}(k)$ defined in (3.6) we must invert the hierarchical matrix $\left(k^{2}+\mu\right) I-\sigma$. Using the algebra on the pairs $\tilde{\sigma}, \sigma(u)$ induced by matrix 
multiplication (see the Appendix II), we see that for each $k, G_{a b}(k)$ is again a hierarchical matrix in replica space, parametrized by a function $g(k, u)(u \in[0,1])$ and the diagonal elements $\tilde{g}(k)$. The relation between the parametrization $\tilde{a}, a(u)$ of a hierarchical matrix $A_{a b}$ and the parametrization $\tilde{b}, b(u)$ of its inverse $B=A^{-1}$ is derived in appendix II. In particular in equation (3.12) we need the combinations :

$$
\tilde{g}(k)-g(k, u)=\frac{1}{u\left(k^{2}+\mu+[\sigma](u)\right)}-\int_{u}^{1} \frac{\mathrm{d} v}{v^{2}} \frac{1}{k^{2}+\mu+[\sigma](v)},
$$

where we have introduced the following transform $[\sigma](u)$ of the function $\sigma(u)$ :

$$
[\sigma](u) \equiv u \sigma(u)-\int_{0}^{u} \sigma(v) \mathrm{d} v .
$$

Using this algebra on can rewrite the stationarity equations (3.12) as :

$$
\sigma(u)=2 \beta \hat{f}^{\prime}\left(\frac{2}{\beta} \int \mathrm{d} k(\tilde{g}(k)-g(k, u))\right),
$$

and the corresponding free energy is (using the expression for the trace of the logarithm of a hierarchical matrix written in appendix II) :

$$
\begin{aligned}
\frac{F}{n N L^{D}}=c^{\mathrm{t}} & +\frac{1}{2 \beta} \int \mathrm{d} k\left(k^{2}+\mu\right) \tilde{g}(k)- \\
& -\frac{\beta}{2} \int_{0}^{1} \mathrm{~d} u \hat{f}\left(\frac{2}{\beta} \int \mathrm{d} k(\tilde{g}(k)-g(k, u))\right)-\frac{1}{2 \beta} \int \mathrm{d} k(\log \{\tilde{g}-\langle g\rangle\}+ \\
& \left.+\frac{g(k, 0)}{\tilde{g}-\langle g\rangle}-\int_{0}^{1} \frac{\mathrm{d} u}{u^{2}} \log \frac{\tilde{g}-\langle g\rangle-[g]}{\tilde{g}-\langle g\rangle}\right),
\end{aligned}
$$

where :

$$
\langle g\rangle \equiv \int_{0}^{1} \mathrm{~d} u g(k, u) .
$$

The above three equations (5.2), (5.3) and (5.4) give a closed functional equation to be solved for the self energy $\sigma(u)$ ( $\tilde{\sigma}$ is then given by (5.1)). These equations are identical, mutatis mutandis to the equations derived by Shakhanovich and Gutin [7], in their study of random heteropolymers.

As we shall see there are two regimes which correspond to qualitatively different functions $\sigma(u)$. These regimes depend on whether $\chi^{\mathrm{F}}\left(\frac{2+\gamma(D-2)}{1+\gamma}\right)$ is positive or negative.

5.2 NOISE WITH LONG RANGE CORRELATIONS. - The first case is $\chi^{\mathrm{F}}>0$. It corresponds to either $D \geqslant 2$ or $D<2$ and $\gamma<\frac{2}{2-D}$. We shall refer to it loosely as the case of long range correlations. It turns out that in this case we may forget the regularization of $f$ at short distances and we can work with (3.9), (3.11):

$$
\hat{f}(x)=\frac{\hat{g}}{2(1-\gamma)} x^{1-\gamma}
$$

The limit $\mu \rightarrow 0$ can also be taken safely in this case. 


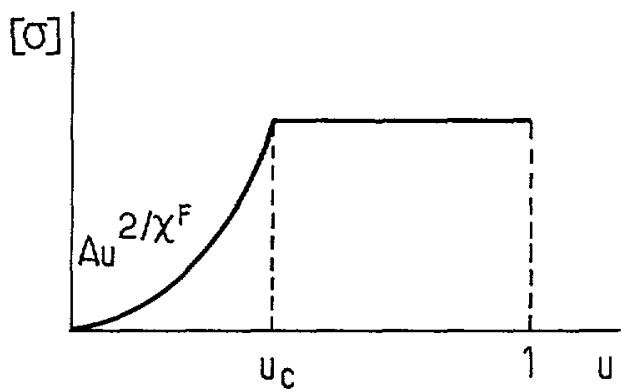

Fig. 3. - The function $[\sigma](u)$ which parametrizes the self energy matrix with broken replica symmetry, in the case where $\chi^{\mathrm{F}}=\frac{2+\gamma(D-2)}{1+\gamma}>0$.

To solve the equations (5.2)-(5.4) for $\sigma$, we differentiate (5.4), which gives $\sigma^{\prime}(u)=0$ or :

$$
\left(2^{-\gamma} \beta^{1+\gamma} \hat{g} \gamma j_{D}([\sigma](u))^{(D-4) / 2}\right)^{1 /(1+\gamma)}=\int \mathrm{d} k(\tilde{g}(k)-g(k, u)),
$$

with $j_{\mathrm{D}}=\Gamma(2-D / 2) /\left(2^{D} \pi^{(D / 2)}\right)$. Differentiating once more gives the following constraint on the self energy function $\sigma(u)$, or rather on its transform $[\sigma](u)$ :

$$
\begin{aligned}
& {[\sigma]^{\prime}(u)=0 \text { or }} \\
& {[\sigma](u)=A u^{2 / x^{F}},}
\end{aligned}
$$

where

$$
A=\left(2^{\frac{1}{\gamma+1}-2} \beta \hat{g}^{\frac{1}{\gamma+1}} j_{D}^{\frac{1}{\gamma+1}-1} \gamma^{\frac{1}{\gamma+1}} \frac{4-D}{\gamma+1}\right)^{2 / x^{\mathrm{F}}}
$$

is a positive constant.

Using (5.8) and (5.9) one can derive the final shape of the $\sigma(u)$ function (see Fig. 3) :

$$
\begin{array}{ll}
{[\sigma](u)=A u^{2 / x^{\mathrm{F}}}} & u<u_{\mathrm{c}}, \\
{[\sigma](u)=A u_{\mathrm{c}}^{2 / x^{\mathrm{F}}}} & u>u_{\mathrm{c}} .
\end{array}
$$

The value of the breakpoint $u_{c}$ depends in general on details of the ultraviolet lattice regularization. In $D<2$ the continuum limit can be taken and one gets :

$$
u_{c}=\frac{(2-D)(1+\gamma)}{4-D} \quad(D<2) \text {. }
$$

In this case of long range correlations we find a solution with a non trivial breaking of replica symmetry. As $[\sigma](u)$ appears like a kind of mass term in the propagator (see (5.2)), the fact that $[\sigma](u)$ extends down to $[\sigma]=0$ (at $u=0$ ) changes the long distance behaviour of the theory.

As we have seen before in (4.4), in order to compute the exponent $\zeta$ we need the diagonal part of the propagator, $G_{a a}(k)=\tilde{g}(k)$. This can be read from appendix II :

$$
\tilde{g}(k)=\frac{1}{k^{2}}\left(1+\int_{0}^{1} \frac{\mathrm{d} u}{u^{2}} \frac{[\sigma](u)}{k^{2}+[\sigma](u)}\right) .
$$


For $\gamma>0$ we use the fact that $\sigma(0)=0$; this relation can be derived from (5.4).

For small $k$ the leading term of $\tilde{g}(k)$ comes from the small $u$ region of the integral; this gives :

$$
\tilde{g}(k) \stackrel{k \rightarrow 0}{\sim} c^{\mathrm{t}}\left(\frac{1}{k^{2}}\right)^{1+2 / x^{\mathrm{F}}},
$$

which corresponds exactly to the Flory result for the exponent $\zeta$ :

$$
\zeta_{\mathrm{RSB}}=\zeta^{\mathrm{F}}=\frac{4-D}{2(1+\gamma)}
$$

Let us briefly recall that this result is valid within the following hypotheses and approximations : $D<4, \gamma>0$ and $\chi^{F}>0$, the variational approximation (3.5) for the free energy, and the hierarchical Ansatz for the replica symmetry breaking. It is also interesting to notice that if the correlation function $\hat{f}$ of the noise is more complicated than a simple power law, the small $u$ behaviour of $[\sigma](u)$, and accordingly the $\zeta$ exponent, are determined by the asymptotic behaviour of $\hat{f}$ (or of $f$ ) at large arguments, which provides the correct definition of the number $\gamma$ in formula (5.15).

5.3 NOISE WITH SHORT RANGE CORRELATIONS. - Let us now turn to the case of short range correlations of the noise where $\chi^{\mathrm{F}}<0$, that is : $D<2$ and $\gamma>\frac{2}{2-D}$. According to the previous section, there exists in this regime a stable replica symmetric solution. On the other hand if we consider the above solution (5.11), we see that when $\chi^{\mathrm{F}}$ goes to zero from above the function $[\sigma](u)$ tends to be flat at the origin while the break point $u_{c}$ tends to one. All this suggests to look for a solution for the self energy with only one step of r.s.b., defined by three parameters :

$$
\begin{aligned}
\sigma(u) & = \begin{cases}\sigma_{0} & u<u_{\mathrm{c}} \\
\sigma_{1} & u>u_{\mathrm{c}}\end{cases} \\
{[\sigma](u) } & =\left\{\begin{array}{cc}
0 & u<u_{\mathrm{c}} \\
u_{\mathrm{c}}\left(\sigma_{1}-\sigma_{0}\right)=\Sigma_{1} & u>u_{\mathrm{c}}
\end{array}\right.
\end{aligned}
$$

The corresponding propagator is :

$$
\begin{aligned}
\tilde{g}(k)-g(k, u) & =\frac{1}{u_{\mathrm{c}}\left(k^{2}+\mu\right)}-\frac{1-u_{\mathrm{c}}}{u_{\mathrm{c}}} \frac{1}{k^{2}+\mu+\Sigma_{1}} \quad\left(u<u_{\mathrm{c}}\right), \\
& =\frac{1}{k^{2}+\mu+\Sigma_{1}} \quad\left(u>u_{\mathrm{c}}\right) . \\
\tilde{g}(k) & =\frac{1}{u_{\mathrm{c}}\left(k^{2}+\mu\right)}-\frac{1-u_{\mathrm{c}}}{u_{\mathrm{c}}} \frac{1}{k^{2}+\mu+\Sigma_{1}}+\frac{\sigma_{0}}{\left(k^{2}+\mu\right)^{2}} .
\end{aligned}
$$

The values of $\sigma_{0}$ and $\sigma_{1}$ are determined from (5.4) and (5.17):

$$
\begin{aligned}
& \sigma_{0}=2 \beta \hat{f}^{\prime}\left(\frac{m-\left(1-u_{\mathrm{c}}\right) S_{1}}{u_{\mathrm{c}}}\right) \stackrel{\mu \rightarrow 0}{=} 0 \\
& \sigma_{1}=2 \beta \hat{f}^{\prime}\left(S_{1}\right),
\end{aligned}
$$


where :

$$
\begin{aligned}
m & \equiv \frac{2 j_{D}}{\beta} \mu \frac{D-2}{2} \\
S_{1} & \equiv \frac{2 j_{D}}{\beta}\left(\mu+\Sigma_{1}\right)^{\frac{D-2}{2}}
\end{aligned}
$$

In order to determine $u_{\mathrm{c}}$ we must first compute the free energy within this one step breaking Ansatz and then write the stationarity equation with respect to $u_{\mathrm{c}}$. The free energy (5.5) is :

$$
\begin{aligned}
\frac{F}{n N L^{D}}=c^{\mathrm{t}}+\frac{1}{2 \beta} \frac{1-u_{\mathrm{c}}}{u_{\mathrm{c}}} \Sigma_{\mathrm{l}} j_{D}(\mu & \left.+\Sigma_{1}\right)^{(D-2) / 2}-\frac{j_{D}}{\beta D} \frac{1-u_{\mathrm{c}}}{u_{\mathrm{c}}}\left(\mu+\Sigma_{1}\right)^{D / 2}+ \\
& +\frac{\beta}{2} u_{\mathrm{c}} \hat{f}\left(\frac{m-\left(1-u_{\mathrm{c}}\right) S_{1}}{u_{\mathrm{c}}}\right)+\frac{\beta}{2}\left(1-u_{\mathrm{c}}\right) \hat{f}\left(S_{\mathrm{l}}\right) .
\end{aligned}
$$

In the limit $\mu \rightarrow 0$ one finally gets the following stationarity equations :

$$
\begin{gathered}
\Sigma_{1}=2 \beta u_{\mathrm{c}} \hat{f}^{\prime}\left(\frac{2 j_{D}}{\beta} \Sigma_{1}^{(D-2) / 2}\right) \\
\left(\frac{2-D}{D}\right) \frac{2 j_{D}}{\beta} \frac{1}{u_{\mathrm{c}}^{2}} \Sigma_{1}^{D / 2}+2 \beta \hat{f}\left(\frac{2 j_{D}}{\beta} \Sigma_{1}^{(D-2) / 2}\right)=0 .
\end{gathered}
$$

In order to get a non trivial solution of these equations we must use the fact that $\hat{f}$ is regularized at small arguments (in this regime the short distance correlation of the noise becomes relevant).

Assuming a behaviour of $\hat{f}(x)$ which is regularized at $x \sim r$ :

$$
\hat{f}(x) \stackrel{x \rightarrow 0}{\sim} \frac{\hat{g}}{2(1-\gamma)}\left(r^{1-\gamma} \hat{f}_{0}+x r^{-\gamma} \hat{f}_{1}+\cdot \quad\right),
$$

one gets after some work the following result : there exists a critical value of $\beta$,

$$
\beta_{c}=\left(\frac{\left|\hat{f}_{0}\right|}{f_{l}^{D / 2}} \frac{D}{(2-D) j_{D}}\right)^{-\frac{2}{2-D}} r^{-\frac{2-2 \gamma+\gamma D}{2-D}},
$$

such that for $\beta<\beta_{\mathrm{c}}$ the only solution is the replica symmetric one.

For $\beta>\beta_{\mathrm{c}}$ there exists a r.s.b. solution with:

$$
\begin{aligned}
& u_{\mathrm{c}}=\left(\frac{\beta}{\beta_{\mathrm{c}}}\right)^{-\frac{2-D}{4-D}} \\
& \sigma_{0}=-\frac{\beta \hat{g}}{2}\left(\frac{j_{D}}{u_{\mathrm{c}}}\right)^{-\gamma} \mu^{\gamma \frac{2-D}{2}} \sim 0 . \\
& \Sigma_{1}=\hat{g} x_{\mathrm{c}} \hat{f}_{1} r^{-\gamma}
\end{aligned}
$$

The same arguments as before show that this solution corresponds to a wandering exponent :

$$
\zeta_{\mathrm{RSB}}=\frac{2-D}{2}
$$


The resulting shape of the $\sigma(u)$ function for this case is shown in figure 4 . We have not checked so far whether there exist other solutions with higher r.s.b. than just this single step breaking. The analysis of the stability of this solution with respect to quadratic fluctuations will be presented elsewhere [23].

The results obtained in this section agree very well with the explicit study $[5,24]$ of directed polymers on a lattice (both on the Bethe tree and in the infinite dimensional limit), where one finds that replica symmetry is spontaneously broken (with a single step r.s.b.) and that equation (5.25) gives the exact value of $\zeta$.

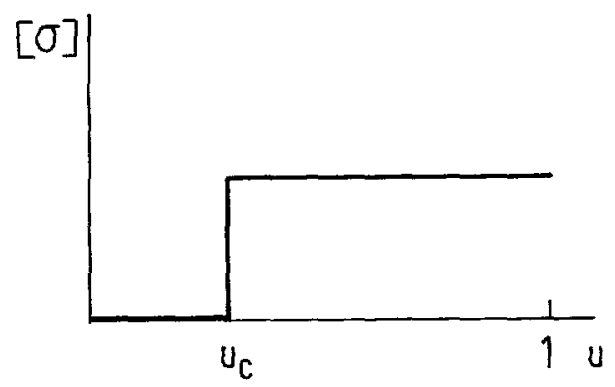

Fig. 4. - The function $[\sigma](u)$ which parametrizes the self energy matrix in the case where $D<2$ and $y<\frac{2}{2-D}$ (" short range " correlation of the noise). There exists then a critical value of the temperature $T_{\mathrm{c}}$. Above $T_{\mathrm{c}}$ the self energy is replica symmetric $([\sigma](u)=0)$. Below $T_{\mathrm{c}}$ there is a one step replica symmetry breaking solution described by the above $[\sigma](u)$ function.

\section{Physical interpretation of replica symmetry breaking.}

In the previous section we have found two types of solutions with r.s.b. for the self energy matrix, summarized in figures 3,4 . These solutions give back the Flory value for the wandering exponent, which is certainly an improvement upon the usual perturbative field theoretic treatment. However these replica computations are rather formal and in this section we want to extract some of the physical information which is hidden in the form of the r.s.b. for the self energy and the propagator. We shall first work out some general features which are encoded in a Gaussian distribution with r.s.b. This is then applied to the two solutions derived before.

6.1 THE GENERAL DECODING OF A GAUSSIAN ULTRAMETRIC REPLICA SYMMETRY BREAKING. - As we keep to our variational Ansatz, we are dealing with free fields with a non trivial (hierarchical) mass matrix. Therefore the basic problem, which is the clue of the physical analysis, can be formulated as follows : let $\omega$ be a $N$ dimensional vector variable (here after it will be for instance one Fourier mode of the interface). We suppose that a physical system is described by a partition function $Z(\omega)$ which is well defined for each $\omega$, but the system contains quenched randomness which leads to fluctuations of $Z(\omega)$ from sample to sample. For one given sample the distribution of $\omega$ is :

$$
P(\omega)=\frac{Z(\omega)}{\int \mathrm{d} \omega^{\prime} Z\left(\omega^{\prime}\right)}
$$


Some of the interesting physical questions concern :

- the averaged (over the quenched randomness) distribution : $\overline{P(\boldsymbol{\omega})}$

- the average joint probabilities: $\overline{P\left(\omega_{1}\right) \cdot \bar{P}\left(\omega_{k}\right)}$

- the distribution of the susceptibility $\chi$ :

$$
\chi=\frac{1}{N}\left(\left\langle\omega^{2}\right\rangle-\langle\omega\rangle^{2}\right)=\frac{1}{N}\left(\int \mathrm{d} \omega P(\omega)\left\langle\omega^{2}\right\rangle-\left(\int \mathrm{d} \omega P(\omega)\langle\omega\rangle\right)^{2}\right) .
$$

Now we suppose that the problem has been studied by the replica method which gives as a result :

$$
\overline{Z\left(\boldsymbol{\omega}_{1}\right) . . Z\left(\boldsymbol{\omega}_{n}\right)}=c^{\mathrm{t}} \sum_{\pi} \exp \left(-\frac{1}{2} \sum_{a, b}\left(Q^{-1}\right)_{\pi(a) \pi(b)} \boldsymbol{\omega}_{a} \cdot \boldsymbol{\omega}_{b}\right),
$$

where the matrix $Q_{a b}$ has a hierarchical (ultrametric) structure in the $n \rightarrow 0$ limit, and the $\sum_{x}$ ranges over all the $n$ ! permutations of the indices $\left({ }^{1}\right)$. The problem is to relate the physical properties of the probability $P(\omega)$ to the matrix $Q$. This is clearly a rather general situation which will appear whenever we treat a random system with the Gaussian Ansatz and ultrametric r.s.b.

The solution of this problem closely follows some developments which took place a few years ago in the mean field theory of spin glasses [13,25]. An important difference is that in the spin glass case one was dealing with Ising variables in place of the vector $\omega$. Most importantly, in the previous analysis of spin glasses the attention was concentrated on the case where an infinite number of variables were present; here we consider also the implications of replica symmetry breaking on the probability distribution of a single variable (or a finite dimensional vector) and we study the new effects which arise in this case. We shall now present some of the results; the proofs are sketched in Appendix III.

Let us first consider the case where $Q$ is a matrix with one step breaking parametrized by $\tilde{q}, q(u)$ such that :

$$
q(u)= \begin{cases}q_{0} & u<u_{\mathrm{c}} \\ q_{1} & u>u_{\mathrm{c}}\end{cases}
$$

Then the physical distribution of $\omega$ is obtained by the following process. For each sample one generates:

- a random variable $\omega_{0}$ distributed as :

$$
\mathfrak{T}\left(\boldsymbol{\omega}_{0}\right)=\frac{1}{\left[\sqrt{2 \pi q_{0}}\right]^{N}} \exp \left(-\frac{\omega_{0}^{2}}{2 q_{0}}\right) .
$$

- «States 》 (we keep to the spin glass terminology), each of which is characterized by a variable $\omega_{\alpha}$ and a weight $W_{\alpha}$. Given $\omega_{0}$, the variables $\omega_{\alpha}$ are uncorrelated. Their distribution is :

$$
\mathcal{T}\left(\boldsymbol{\omega}_{1}, \omega_{2}, . .\right)=\prod_{\alpha} \frac{1}{\left[\sqrt{2 \pi\left(q_{1}-q_{0}\right)}\right]^{N}} \exp \left(-\frac{\left(\omega_{\alpha}-\omega_{0}\right)^{2}}{2\left(q_{1}-q_{0}\right)}\right)
$$

(') In the previous section, it is clear that whenever one finds a solution $\sigma_{a b}$ of the stationarity equations (3.12), the matrix $\sigma_{a b}^{\pi}=\sigma_{\pi(a) \pi(b)}$ is also a solution for any permutation $\pi$. When there is r.s.b., this generates new solutions, with the same free energy. One should then sum over all such solutions, as in (6.3). 
The weights $W_{\alpha}$ are derived from some «free energy " variables $f_{\alpha}$ through :

$$
W_{\alpha}=\frac{\mathrm{e}^{-\beta f_{\alpha}}}{\sum_{\nu} \mathrm{e}^{-\beta f_{\nu}}}
$$

The $f_{\alpha}$ are independent random variables with an exponential distribution such that the average number of states with free energy less than $f$ (i.e. weight $W_{\alpha}$ greater than $\left.\mathrm{e}^{-\beta f}\right)$ is :

$$
\mathcal{N}(f) \sim \mathrm{e}^{\rho\left(f-f_{\mathrm{s}}\right)}
$$

where $\rho=\beta u_{\mathrm{c}}$ and $f_{\mathrm{s}}$ is an arbitrary scale. (A more precise mathematical definition using Poisson's process can be found in [26]).

Eventually, after the "states" have been generated for one sample, we have the probability distribution of $\omega$ inside this sample:

$$
P(\omega)=\sum_{\alpha} W_{\alpha} \frac{1}{\left[\sqrt{\left.2 \pi\left(\tilde{q}-q_{1}\right)\right]^{N}}\right.} \exp \left(-\frac{\left(\omega-\omega_{\alpha}\right)^{2}}{2\left(\tilde{q}-q_{1}\right)}\right) .
$$

If we go to two steps of r.s.b. (with $q(u)$ taking the values $q_{0}, q_{1}, q_{2}$, respectively when $u$ lies in the intervals $\left[0, u_{1}\right],\left[u_{1}, u_{2}\right],\left[u_{2}, u_{3}\right]$ ), there is one more generation (the "clusters $\left."\right)$ in the ultrametric tree and the physical distribution is generated for each sample according to the following steps:

- generate $\omega_{0}$ as before ;

- generate «clusters» $\left(\omega_{\mathrm{c}}, F_{\mathrm{c}}\right)$ such that (the notations are obvious generalizations of the ones above):

$$
\mathfrak{S}\left(\left\{\boldsymbol{\omega}_{\mathrm{c}}\right\}\right)=\prod_{\mathrm{c}} \frac{1}{\left[\sqrt{\left.2 \pi\left(q_{1}-q_{0}\right)\right]^{N}}\right.} \exp \left(-\frac{\left(\boldsymbol{\omega}_{\mathrm{c}}-\boldsymbol{\omega}_{0}\right)^{2}}{2\left(q_{1}-q_{0}\right)}\right),
$$

and the average number of clusters with free energy $F_{\mathrm{c}}=F$ is :

$$
\mathcal{N}(F)=\mathrm{e}^{\beta u_{1}\left(F-F_{s}\right)},
$$

— within each «cluster » $\omega_{c}, F_{c}$, generate « states» $\left(\omega_{\alpha}, f_{\alpha}\right)$ such that :

$$
\mathfrak{T}\left(\boldsymbol{\omega}_{\alpha}\right)=\frac{1}{\left[\sqrt{\left.2 \pi\left(q_{2}-q_{1}\right)\right]^{N}}\right.} \exp \left(-\frac{\left(\omega_{\alpha}-\omega_{c}\right)^{2}}{2\left(q_{2}-q_{1}\right)}\right)
$$

their average number at free energy $f$ is :

$$
\mathcal{N}(f)=\mathrm{e}^{\beta u_{2}\left(f-F_{\mathrm{c}}\right)}
$$

Eventually, the distribution of $\omega$ for the sample so generated is :

$$
P(\boldsymbol{\omega})=\sum_{\alpha}\left(\frac{\mathrm{e}^{-\beta f_{\alpha}}}{\sum_{\nu} \mathrm{e}^{-\beta f_{\nu}}}\right) \frac{1}{\left[\sqrt{2 \pi\left(\tilde{q}-q_{2}\right)}\right]^{N}} \exp \left(-\frac{\left(\omega-\omega_{\alpha}\right)^{2}}{2\left(\tilde{q}-q_{2}\right)}\right) .
$$


The process is then iterated in an obvious hierarchical way (clusters within clusters) when the matrix $Q$ is parametrized by a higher order r.s.b. In order to study the limit of a matrix $Q$ parametrized by a more complicated function $q(u)$, one must take the continuum limit of the above process (as the limit of an infinite number of breakings). A precise mathematical definition of this limit has been worked out in [26].

To conclude this section let us mention some aspects of the distribution of the susceptibility. The average susceptibility is :

$$
\bar{x}=\frac{1}{N}\left(\overline{\left\langle\omega^{2}\right\rangle}-\overline{\langle\omega\rangle^{2}}\right)=\int_{0}^{1} \mathrm{~d} u(\tilde{q}-q(u)) .
$$

The sample to sample fluctuations of the susceptibility is derived more easily in the large $N$ limit. One gets for instance :

$$
\bar{\chi}_{\overparen{N \rightarrow \infty}}^{2} \frac{2}{3}\left(\int_{0}^{1} \mathrm{~d} u(\tilde{q}-q(u))\right)^{2}+\frac{1}{3} \int_{0}^{1} \mathrm{~d} u(\tilde{q}-q(u))^{2} .
$$

From these two formulas it is clear that the existence of sample to sample fluctuations of the susceptibility is related to replica symmetry breaking. When such fluctuations are present it would be interesting to know the typical values of the susceptibility rather than just the first moments of the distribution; this is more complicated and we leave it for future work. A related quantity concerns the distribution of $\boldsymbol{\omega}$.

From the above physical description (or in a more compact way from the replica representation (5.3)) one deduces

$$
\begin{gathered}
\overline{P(\boldsymbol{\omega})}=\frac{1}{\left[\sqrt{2 \pi \tilde{q}]^{N}}\right.} \exp \left(-\frac{1}{2} \frac{\boldsymbol{\omega}^{2}}{2 \tilde{q}}\right), \\
\overline{P(\boldsymbol{\omega}) P\left(\boldsymbol{\omega}^{\prime}\right)}=\int_{0}^{1} \frac{\mathrm{d} u}{\left[\sqrt{\left.2 \pi\left(\tilde{q}^{2}-q(u)^{2}\right)\right]^{N}}\right.} \exp \left(-\frac{1}{2}\left\{\frac{\tilde{q}^{2}\left(\boldsymbol{\omega}^{2}+\boldsymbol{\omega}^{\prime 2}\right)-2 q(u) \boldsymbol{\omega} \cdot \boldsymbol{\omega}^{\prime}}{\tilde{q}^{2}-q(u)^{2}}\right\}\right) .
\end{gathered}
$$

This last formula is of special interest for the following reason: let us consider two fields $\omega$ and $\omega^{\prime}$ in the same random environment. The average of the probability distribution of the difference $\Delta=\omega-\omega^{\prime}$ is :

$$
\begin{aligned}
\overline{P(\Delta)} & =\int \mathrm{d} \boldsymbol{\omega} \mathrm{d} \boldsymbol{\omega}^{\prime} \overline{P(\boldsymbol{\omega}) P\left(\omega^{\prime}\right)} \delta\left(\boldsymbol{\omega}-\boldsymbol{\omega}^{\prime}-\Delta\right) \\
& =\int_{0}^{1} \frac{\mathrm{d} u}{\left[\sqrt{4 \pi(\tilde{q}-q(u))]^{N}}\right.} \exp \left(-\frac{1}{2} \frac{\Delta^{2}}{2(\tilde{q}-q(u))}\right) .
\end{aligned}
$$

6.2 THE CASE OF «SHORT RANGE 》 CORRELATIONS OF THE NOISE (ONE STEP r.s.b.). - As we saw in the previous section, the self energy matrix is described by a one step r.s.b. function when $D<2$ and $\gamma>\frac{2}{D-2}$, at low enough temperatures. The Fourier modes of the $\omega$ fields have the following distribution :

$$
c^{\mathrm{t}} \exp \left(-\frac{\beta}{2} \sum_{a, b} \int \mathrm{d} k\left(k^{2} \delta_{a b}-\sigma_{a b}\right) \omega_{a}(k) \cdot \omega_{b}(k)\right) .
$$


The previous analysis can be applied separately for each Fourier mode since they are uncoupled.

For each mode with wavevector $k$ we first compute $Q_{a b}=(1 / \beta)\left(\left(k^{2} I-\sigma\right)^{-1}\right)_{a b}=$ $(1 / \beta) G_{a b}(k)$. This propagator has been studied at length in the previous section; it is parametrized by $\tilde{q}=\tilde{g}(k), q(u)=g(k, u)$, where the expressions for $\tilde{g}$ and $g$ have been derived in (5.2) and (5.13). We thus have :

$$
\begin{aligned}
& q_{0}=0 \\
& q_{1}=\frac{1}{\beta u_{\mathrm{c}}}\left(\frac{1}{k^{2}}-\frac{1}{k^{2}+\Sigma_{1}}\right)=\frac{\Sigma_{1}}{\beta u_{\mathrm{c}}} \frac{1}{k^{2}\left(k^{2}+\Sigma_{1}\right)} \\
& \tilde{q}(k)=q_{1}+\frac{1}{\beta\left(k^{2}+\Sigma_{1}\right)},
\end{aligned}
$$

where $\Sigma_{1}$ and $u_{c}$ have been obtained in (V.24).

As $q_{0}=0$ we have $\boldsymbol{\omega}_{0}=\mathbf{0}$. For each sample we generate directly the «states» $\alpha$ with :

$$
\mathfrak{T}\left(\left\{\boldsymbol{\omega}_{\alpha}\right\}\right)=\prod_{k, \alpha}\left[\sqrt{\frac{\beta u_{\mathrm{c}} k^{2}\left(k^{2}+\Sigma_{1}\right)}{2 \pi \Sigma_{1}}}\right]^{N} \exp \left(-\frac{1}{2} \omega_{\alpha}(k) \cdot \omega_{\alpha}(-k) \frac{\beta u_{\mathrm{c}}}{\Sigma_{1}} k^{2}\left(k^{2}+\Sigma_{1}\right)\right)
$$

and weights $P_{\alpha}$ with the exponential distribution (6.7). Then :

$$
\begin{aligned}
P(\omega)=\prod_{k}\left[\sqrt{\frac{\beta\left(k^{2}+\Sigma_{1}\right)}{2 \pi}}\right]^{N} \sum_{\alpha} W_{\alpha} \times \\
\\
\quad \times \exp \left(-\frac{1}{2}\left(\omega(k)-\omega_{\alpha}(k)\right) \cdot\left(\omega(-k)-\omega_{\alpha}(-k)\right) \beta\left(k^{2}+\Sigma_{1}\right)\right) .
\end{aligned}
$$

If we concentrate on the large scale fluctuations (small $|k|$ ), we see that the fluctuations of $\omega$ around each "state $" \omega_{\alpha}$ remain bounded (because of the presence of the mass term $\Sigma_{1}$ in (6.22)). The leading fluctuations are due to the large lateral extension which can be reached by each state $\omega_{\alpha}$. on a scale of longitudinal dimension $|x|$, we have from (6.21)

$$
\left.\overline{\left(\omega_{\alpha}(x)-\omega_{\alpha}(0)\right.}\right)^{2} \sim \int \mathrm{d}^{D} k \frac{1-\cos (k \cdot x)}{\frac{u \beta_{\mathrm{c}}}{\Sigma_{1}} k^{2}\left(k^{2}+\Sigma_{1}\right)} \sim c^{\mathfrak{t}}|x|^{2-D},
$$

which leads to the exponent $\zeta=\frac{2-D}{2}$ as derived before.

However we see here that the physics of this one step r.s.b. solution is quite different from that of the replica symmetric one $(\sigma(u)=0)$, although both give the same $\zeta$ : the whole structure of «states» is absent in the replica symmetric solution.

If we concentrate on the directed polymer case $\left(D=1, \gamma=1+\frac{N}{2}\right)$, we see that the above one step r.s.b. is supposed to apply whenever $N>N_{c}=2$ (within the Gaussian approximation only - there is no reason to believe that this value of $N_{\mathrm{c}}$ will persist beyond the Gaussian approximation), and it is supposed to be exact when $N \rightarrow \infty$. This seems to 
agree with direct studies of the polymer problem on the Bethe lattice, and $\frac{1}{N}$ expansions around this kind of $N=\infty$ limit, which gave precisely the same picture in which there is only one generation of «states». The above distribution $P(\boldsymbol{\omega})$ in $(6.22)$ provides a precise generalization of this mean field picture to finite dimensional systems. It would be interesting to be able to test it numerically, but this seems quite difficult.

6.3 THE CASE OF FULL r.s.b. - We now turn to the case where $\chi^{\mathrm{F}}$ is positive, so that the self energy matrix is described by a non constant $\sigma(u)$ function. The decoupling of the various Fourier modes (Eq. (6.19)) still holds, but the picture of states within clusters within clusters etc... is more subtle because the continuum limit of r.s.b. has to be taken.

A simpler problem consists in studying the distribution of $\boldsymbol{\omega}$ defined as:

where $|x| \gg a$.

$$
\omega=\omega(x)-\omega(0)
$$

In replica space the $\omega_{a}$ (which are linear combinations of Gaussian variables) are Gaussian. Their distribution is of the general form (6.3), where :

$$
Q_{a b}=\frac{2}{\beta} \int \mathrm{d} k G_{a b}(k)(1-\cos (k \cdot x)) .
$$

$Q_{a b}$ is parametrized by $\tilde{q}, q(u)$ which can be computed from the expressions derived previously (5.2), (5.11) and (5.13). For large $|x|$, simple scaling analysis of these expressions shows that :

$$
\begin{aligned}
& \tilde{q} \sim|x|^{2 \zeta^{F}}, \\
& \tilde{q}-q(u) \sim\left\{\begin{array}{lll}
u^{-2 \xi / x^{\mathrm{F}}} & \text { if } u>|x|^{-\chi^{\mathrm{F}}} \\
|x|^{2 x^{\mathrm{F}}} & \text { if } u<|x|^{-\chi^{\mathrm{F}}}
\end{array}\right.
\end{aligned}
$$

Because one is studying the problem on a finite length scale $|x|$, the singular behaviour of $q(u)$ at small $u$ is cut-off. The largest clusters $\left(\omega_{\mathrm{c}}, F_{\mathrm{c}}\right)$ dominate the fluctuations on this scale. They have variables $\left|\omega_{c}\right|$ or order $|x|^{\zeta}$, and their free energy distribution is exponential (6.8) with $\rho=|x|^{-\chi^{\mathrm{F}}}$, which means that their typical free energy differences are of order $|x|^{x^{\mathrm{F}}}$. This provides the scale of the free energy fluctuations for a sample of size $|x|$, which is reasonable.

As for the distribution of the susceptibility we deduce from the general analysis (6.15):

$$
\bar{\chi}=2 \int_{0}^{1} \mathrm{~d} u \int \mathrm{d} k(\tilde{g}(k)-g(k, u))(1-\cos (k \cdot x)) \text {. }
$$

Using the result :

$$
\int_{0}^{1} \mathrm{~d} u(\tilde{g}(k)-g(k, u))=\frac{1}{k^{2}}
$$

(this is nothing but the result of the sum rule $\sum_{b} \sigma_{a b}=0$, which can also be understood as the result of the Ward identity expressing translational invariance), we get:

$$
\bar{\chi} \underset{|x|>a}{ } c^{t}|x|^{(2-D)}=c^{t}|x|^{2 \xi-x^{F}}
$$


The second moment of $\chi, \bar{\chi}^{2}$, is expressed in (6.16). For large $|x|$, it is dominated by the second term in (6.16) which gives :

$$
\bar{\chi}^{2} \underset{|x| \geqslant a}{ } c^{t}|x|^{2(2-D)+\chi^{F}}=c^{t}|x|^{2(2 \zeta)-x^{F}}
$$

For higher moments one can show that, for large $|x|$ the leading term in $\bar{x}^{k}$ is the one of the form $\int_{0}^{1} \mathrm{~d} u(\tilde{q}-q(u))^{k}$. This gives :

$$
\bar{\chi}^{k} \sim c_{k}|x|^{k(2 \zeta)-x^{F}}
$$

The interpretation of this formula is that the integer moments of the $\chi$ distribution at a large scale $|x|=\ell$ are dominated by rare samples: one sample every $\ell^{-} \chi^{F}$ will be such that two of the clusters free energies $F_{c}$ and $F_{c^{\prime}}$ will be essentially equal (i.e. they differ by terms of order 1, while the typical spacing of $F_{c}$ 's are of order $\ell^{F}$ ). For these samples the susceptibility is of order $\left|\omega_{c}-\omega_{c^{\prime}}\right|^{2}$. As $\left|\omega_{c}\right| \sim \ell^{\zeta}$, we are led to (6.31). This effect agrees with previous studies : it has been analyzed first on the case of directed polymers [15, 27]. The existence of a relation between exponents leading to the simple result (6.31) for the average susceptibility has been studied for general manifolds in [28].

Instead of the typical value of the susceptibility, we now study a quantity which is somewhat related to it, i.e. the distribution of $\Delta=\omega-\omega^{\prime}$ where $\omega(=\omega(x)-\omega(0))$ and $\omega^{\prime}\left(=\omega^{\prime}(x)-\omega^{\prime}(0)\right)$ are two manifolds in the same random potential. When we substitute the actual form of $\tilde{q}-q(u)$ in (6.26), with its cut off at $u \sim|x|^{-x^{\mathrm{F}}}$, into the general distribution $P(\Delta)$ in $(6.18)$, we find two kinds of terms :

- The contribution to the integral coming from the small $u$ region $\left(u \leqslant|x|^{-\chi^{\mathrm{F}}}\right)$ contributes to $P(\boldsymbol{\Delta})$ as :

$$
P_{1}(\Delta) \sim|x|^{-x^{F}-\zeta N} \exp \left(-\frac{\Delta^{2}}{2|x|^{2 \zeta}}\right)
$$

which corresponds to the rare samples (dominating the integer moments of the distribution) described before for the susceptibility.

- The rest of the integral gives:

$$
P_{2}(\Delta) \sim \int_{A|x|-x^{\mathrm{F}}}^{1} \mathrm{~d} u u^{\frac{\zeta N}{x^{\mathrm{F}}}} \exp \left(-\frac{\Delta^{2}}{2 u^{-2 \zeta / x^{\mathrm{F}}}}\right) .
$$

The last term is the distribution of $\Delta$ for the generic samples. It gives a $P_{2}(\Delta)$ which is finite at $\boldsymbol{\Delta}=\mathbf{0}$ and decreases like a power law at large $|\boldsymbol{\Delta}|$ :

$$
P_{2}(\Delta) \overbrace{1 \ll|\Delta| \ll|x|^{2 \zeta}}|\Delta|^{-N-\frac{x^{\mathrm{F}}}{2 \zeta}}
$$

Therefore the typical values of $|\mathbf{\Delta}|$ are finite $(|x|$ independent). Actually this slow power law fall off of the distribution of $\boldsymbol{\Delta}$ reproduces the scaling $\left(P(\boldsymbol{\Delta}) \sim|x|^{-\omega^{\mathrm{F}}}\right)$ for $|\boldsymbol{\Delta}| \sim|x|^{2 \zeta}$ as seen before. This tail is therefore at the origin of the importance of rare samples on integer moments of $\Delta$. 
As we have already mentioned, the physical conclusions which can be drawn from the full r.s.b. solution of figure 4 are in good agreement with what is known for the directed polymer case, e.g. in the case $D=1, N=1, \gamma=3 / 2$. The main mismatch lies in the value of the exponent, since the r.s.b. solution gives in this case $\zeta=\zeta^{F}=3 / 5$ while the correct exponent is $\zeta=2 / 3$. Whether the expansions of the free energy beyond the Gaussian approximation will help to correct $\zeta$ is an interesting open question. In this respect let us just remind that it has been argued, using the Bethe Ansatz method, that replica symmetry should be broken only in a weak sense (that means only when an actual coupling between two copies of the system is introduced). Within the variational approach we find a stronger breaking, with a numerical result for $\zeta$ which is not correct, but with apparently the correct physical picture of the system.

\section{Discussion.}

There is little doubt that most of the physical properties of manifolds in random media are intimately related to the existence of many configurations of the manifold which are locally optimal and have a low free energy. While usual perturbation theory is inappropriate in such a context, replica symmetry breaking provides a formalism in which these low lying metastable states can be taken can of in a very easy way.

In order to give a relatively simple example, let us consider only the case of directed polymers $(D=1)$. The original problem may be formulated in a slightly different way. We consider the stochastic differential equation :

$$
\frac{\partial Z(t, x)}{\partial t}=\nabla^{2} Z(t, x)+V(t, x) Z(t, x)
$$

where $V$ is the random potential, and one imposes initial conditions at $t=0$ (the most popular ones are a) $Z(0, x)=1$ or b) $Z(0, x)=\delta(x)$ ) ; at some stage it may be convenient to restrict the equation to a transverse box $|x|<R$. The function $Z$, which depends on the sample (i.e. the choice of the random potential $V$ ), allows to compute various expectations values of $x$ at time $t$, like :

$$
\left\langle x^{\mu_{1}} \quad x^{\mu_{k}}\right\rangle_{t}=\int \mathrm{d} x x^{\mu_{1}} \cdot x^{\mu_{k}} \psi(x, t),
$$

where :

$$
\psi(x, t)=\frac{Z(x, t)}{\int \mathrm{d} y Z(y, t)}
$$

The problem is to find the probability distribution of $\psi(x, t)$ at a fixed time $t$. In other words we must find a functional $\mathfrak{T}_{t}[\phi]$ such that :

$$
\overline{F[\psi(x, t)}]=\int \mathrm{d}[\phi] \mathfrak{F}_{t}[\phi] F[\phi],
$$

where $F$ is a generic functional of $\psi(x, t)$ (for $x$ belonging to the whole domain, but fixed $t$ ), and $d[\phi]$ denotes functional integration. 
For practical purposes we are interested in approximated forms of the functional such that the functional integral at the r.h.s. of equation (7.4) can be evaluated. For example in one dimension we could write :

$$
\mathfrak{T}_{t}[\phi]=\exp -\left(\int \mathrm{d} x\left(\frac{\partial \ln \phi}{\partial x}\right)^{2}\right) .
$$

It can be proven that this representation becomes exact in the large time limit, if the noise has a delta function correlation (white noise). This is a rather fortunate case because we obtain a Gaussian probability distribution for the logarithm of the function $\psi$ and many expectations values may be evaluated analytically.

In the generic case, it is not easy to find out reasonable simple approximations for the functions $\mathfrak{T}_{t}[\phi]$, apart from the Gaussian case. The formalism of broken replica symmetry provides a large variety of functionals $\mathcal{S}_{t}[\phi]$ which have the following properties :

a) They depend on many parameters so that we have a large variety of choices.

b) If we want, we can construct these probabilities in such a way that the system is scaling invariant at large distances.

c) The expectation values with respect to the probability $\mathfrak{I}_{t}[\phi]$ can be computed explicitly so that it can be taken as the starting point of a perturbative expansion.

d) Last, but not least, the r.s.b. Ansatz for $\mathfrak{T}_{\imath}[\phi]$ becomes exact when the dimension of the space goes to infinity.

It seems to us that the formalism of broken replica symmetry captures most of the relevant features of the models we consider and it should be a good starting point for more refined approximations.

\section{Appendix I.}

In the representation (3.15), there exists for large $N$ a uniform saddle point :

$$
\begin{aligned}
& r_{a b}(x)=\rho_{a b} \\
& s_{a b}(x)=\sigma_{a b},
\end{aligned}
$$

where $\sigma$ satisfies equation (3.12) with $f$ replaced by $\hat{f}$ and

$$
\rho_{a b}=\int \mathrm{d} k\left[\left(k^{2} I-\sigma\right)^{-1}\right]_{a b} .
$$

In order to lighten the notations, in this appendix we have not written explicitely the $\mu$ terms. All the propagators appearing hereafter are implicitely regularized at small momentum by a small mass term as in (3.6).

In this Appendix we compute the quadratic form of the fluctuations around the saddle point, which allows the study of the stability of various saddle points and is the starting point of a $1 / N$ expansion. We write :

$$
\begin{aligned}
& r_{a b}(x)=\rho_{a b}+\delta r_{a b}(x) \\
& s_{a b}(x)=\sigma_{a b}+\delta s_{a b}(x)
\end{aligned}
$$

and we expand $G$ (defined in (3.16)) to second order in $\delta r$ and $\delta s$ : 


$$
\begin{aligned}
G[r, s]=G[\rho, \sigma]-\frac{\beta}{2} \sum_{a, b} \int \mathrm{d} k \delta r_{a b}(k) \delta s_{a b}(-k)+ \\
\quad-\frac{\beta^{2}}{4} \sum_{a, b} f^{\prime \prime}\left(\rho_{a a}+\rho_{b b}-2 \rho_{a b}\right) \int \mathrm{d} k\left(\delta r_{a a}(k)+\delta r_{b b}(k)-2 \delta r_{a b}(k)\right) \times \\
\quad \times\left(\delta r_{a a}(-k)+\delta r_{b b}(-k)-2 \delta r_{a b}(-k)\right)+\frac{\beta^{2}}{8} \sum_{a b, c d} \int \mathrm{d} k \mathrm{~d} k^{\prime} \delta s_{a b}(k) \delta s_{c d}(-k) \\
{\left[\left(\frac{1}{k^{\prime 2}-\sigma}\right)_{a c}\left(\frac{1}{\left(k-k^{\prime}\right)^{2}-\sigma}\right)_{b d}+\left(\frac{1}{k^{\prime 2}-\sigma}\right)_{a d}\left(\frac{1}{\left(k-k^{\prime}\right)^{2}-\sigma}\right)_{b c}\right] }
\end{aligned}
$$

(we use a loose notation where $\delta s_{a b}(k)$ and $\delta r_{a b}(k)$ are the Fourier transforms of $\delta s_{a b}(x)$ and $\delta r_{a b}(x)$ respectively).

It is convenient to introduce in the place of the $\delta r_{a b}(k),(a<b)$, new fields $X_{a b}$ defined as :

$$
X_{a b}(k)=\frac{\delta r_{a a}(k)+\delta r_{b b}(k)}{2}-\delta r_{a b}(k) \quad(a<b) .
$$

When computing in the Gaussian approximation:

$$
\bar{Z}^{\dot{n}} \sim \int \prod_{a \leq b} d\left[\delta r_{a b}\right] d\left[\delta s_{a b}\right] \mathrm{e}^{N G}=\int \prod_{a} d\left[\delta r_{a a}\right] d\left[\delta s_{a a}\right] \prod_{a<b} d\left[X_{a b}\right] d\left[\delta s_{a b}\right] \mathrm{e}^{N G},
$$

we can perform explicitly the linear integral over $\delta r_{a a}$, which constrains the $\delta s$ field to the subspace :

$$
\delta s_{a a}=-\sum_{b(\neq a)} \delta s_{a b} .
$$

The diagonal Gaussian integral over the $X_{a b}$ can also be performed explicitly, so that we are left eventually with :

$$
\begin{aligned}
\check{Z^{n}} & \sim \int \prod_{a<b} d\left[\delta s_{a b}\right] \exp \left(\frac{N}{2} \sum_{a<b} \frac{1}{f^{\prime \prime}\left(\rho_{a a}+\rho_{b b}-2 \rho_{a b}\right)} \int \mathrm{d} k \delta s_{a b}(k) \delta s_{a b}(-k)+\frac{N \beta^{2}}{4} \times\right. \\
& \times \sum_{a<b} \sum_{c<d} \int \mathrm{d} k \int \mathrm{d} k^{\prime}\left[\left(\frac{1}{k^{\prime 2}+\sigma}\right)_{a c}+\left(\frac{1}{k^{\prime 2}+\sigma}\right)_{b d}-\left(\frac{1}{k^{\prime 2}+\sigma}\right)_{a d}-\left(\frac{1}{k^{\prime 2}+\sigma}\right)_{b c}\right] \\
& \times\left[\left(\frac{1}{\left(k-k^{\prime}\right)^{2}}+\sigma\right)_{a c}+\left(\frac{1}{\left(k-k^{\prime}\right)^{2}+\sigma}\right)_{b d}-\left(\frac{1}{\left(k-k^{\prime}\right)^{2}+\sigma}\right)_{a d}\right. \\
& \left.\left.-\left(\frac{1}{\left(k-k^{\prime}\right)^{2}+\sigma}\right)_{b c}\right] \delta s_{a b}(k) \delta s_{c d}(-k)\right) .
\end{aligned}
$$

Defining further :

$$
u_{a b}(k)=\frac{\sqrt{N} \delta s_{a b}(x)}{\sqrt{\left|f^{\prime \prime}\left(\rho_{a a}+\rho_{b b}-2 \rho_{a b}\right)\right|}}
$$

(we suppose that $f^{\prime \prime}\left(\rho_{a a}+\rho_{b b}-2 \rho_{a b}\right)$ is negative on the saddle point), we have :

$$
\overline{Z^{n}} \sim \int \prod_{a<b} d\left[u_{a b}\right] \exp \left(-\frac{1}{2} \sum_{a<b, c<d} \int \mathrm{d} k M_{a b, c d}(k) u_{a b}(k) u_{c d}(-k)\right),
$$


where :

$$
\begin{aligned}
M_{a b, c d}(k) & =\delta_{(a b),(c d)}-\frac{\beta^{2}}{2} \sqrt{\left|f^{\prime \prime}\left(\rho_{a a}+\rho_{b b}-2 \rho_{a b}\right)\right|\left|f^{\prime \prime}\left(\rho_{c c}^{\prime}+\rho_{d d}-2 \rho_{c d}\right)\right|} \times \\
\times & \int \mathrm{d} k^{\prime}\left[\left(\frac{1}{k^{\prime 2}+\sigma}\right)_{a c}+\left(\frac{1}{k^{\prime 2}+\sigma}\right)_{b d}-\left(\frac{1}{k^{\prime 2}+\sigma}\right)_{a d}-\left(\frac{1}{k^{\prime 2}+\sigma}\right)_{b c}\right] \\
\times & {\left[\left(\frac{1}{\left(k-k^{\prime}\right)^{2}+\sigma}\right)_{a c}+\left(\frac{1}{\left(k-k^{\prime}\right)^{2}+\sigma}\right)_{b d}\right.} \\
& \left.-\left(\frac{1}{\left(k-k^{\prime}\right)^{2}+\sigma}\right)_{a d}-\left(\frac{1}{\left(k-k^{\prime}\right)^{2}+\sigma}\right)_{b c}\right] .
\end{aligned}
$$

A given saddle point $\rho, \sigma$ is stable (respectively marginally stable) if and only if all the eigenvalues of the matrices $M_{a b, c d}(k)$ (for all $k$ 's) are strictly positive (respectively $\geqslant 0$ ).

\section{Appendix II.}

We compute the inverse of a hierarchical matrix $A$, as well as $\operatorname{Tr} \log A$. We suppose that the hierarchical matrix $A_{a b}$ (where $a, b \in\{1, \ldots, n\}$ ) is parametrized by the function $a(u)(u \in[0,1])$ and the diagonal element $A_{a a}=\tilde{a}$.

The algebra of hierarchical matrices has been worked out in [29]. Taking two such matrices $A$, parametrized by $a(u), \tilde{a}$ and $B$, parametrized by $b(u), \tilde{b}$, the product $C=A B$ is parametrized by $c(u), \tilde{c}$, where :

$$
\begin{aligned}
\tilde{c} & =\tilde{a} \tilde{b}-\langle a b\rangle \\
c(u) & =(\tilde{b}-\langle b\rangle) a(u)+(\tilde{a}-\langle a\rangle) b(u)-\int_{0}^{u} \mathrm{~d} v(a(u)-a(v))(b(u)-b(v)),
\end{aligned}
$$

where :

$$
\langle a\rangle=\int_{0}^{1} \mathrm{~d} u a(u) .
$$

In order for $B$ to be the inverse of $A$ we need that in the above equations $\tilde{c}=1$ and $c(u)=0$.

It is convenient to associate to each function $a(u)$ on $[0,1]$ the function $[a]$ defined on the same interval by:

$$
[a](u) \equiv-\int_{0}^{u} \mathrm{~d} v a(v)+u a(u)
$$

From (AII.1) we find that $B=A^{-1}$ implies :

$$
\tilde{a} \tilde{b}-\langle a b\rangle=1
$$

$\forall u \in[0,1]$

$$
(\tilde{a}-\langle a\rangle-[a](u))(\tilde{b}-\langle b\rangle-[b](u))=1 .
$$

Differentiating once (AII.1) and using (AII.4) we get :

$$
b(u)-b(v)=\int_{u}^{v} \mathrm{~d} y a^{\prime}(y)\left(\frac{1}{\tilde{a}-\langle a\rangle-[a](y)}\right)^{2}
$$


Using (AII.1) again we obtain the final result :

$$
\begin{aligned}
& b(u)=-\frac{1}{\tilde{a}-\langle a\rangle} \times \\
& \times\left(\frac{[a](u)}{u(\tilde{a}-\langle a\rangle-[a](u))}+\int_{0}^{u} \frac{\mathrm{d} v}{v^{2}} \frac{[a](v)}{\tilde{a}-\langle a\rangle-[a](v)}+\frac{a(0)}{\tilde{a}-\langle a\rangle}\right) . \\
& \tilde{b}=\frac{1}{\tilde{a}-\langle a\rangle}\left(i-\int_{0}^{1} \frac{\mathrm{d} u}{u^{2}} \frac{[a](u)}{\tilde{a}-\langle a\rangle-[a](u)}-\frac{a(0)}{\tilde{a}-\langle a\rangle}\right) .
\end{aligned}
$$

Let us also mention for completeness three useful formulas :

$$
\begin{gathered}
\tilde{b}-b(u)=\frac{1}{u(\tilde{a}-\langle a\rangle-[a](u))}-\int_{u}^{1} \frac{\mathrm{d} v}{v^{2}} \frac{1}{\tilde{a}-\langle a\rangle-[a](v)} \quad(u>0) . \\
\tilde{b}-b(u)=\frac{1}{\tilde{a}-a(1)}-\int_{u}^{1} \mathrm{~d} v a^{\prime}(v)\left(\frac{1}{\tilde{a}-\langle a\rangle-[a](v)}\right)^{2} \\
\langle b\rangle=-\frac{1}{\tilde{a}-\langle a\rangle}\left(\int_{0}^{1} \frac{\mathrm{d} u}{u^{2}} \frac{[a](u)}{\tilde{a}-\langle a\rangle-[a](u)}+\frac{a(0)}{\tilde{a}-\langle a\rangle}\right) .
\end{gathered}
$$

Let us now turn to the computation of $\operatorname{Tr} \log A$. This quantity can be deduced from the computations performed in [30], formulas (13-15). After some work one gets :

$$
\frac{1}{n} \operatorname{Tr} \log A \underset{n \rightarrow 0}{\sim} \log (\tilde{a}-\langle a\rangle)+\frac{a(0)}{\tilde{a}-\langle a\rangle}-\int_{0}^{1} \frac{\mathrm{d} u}{u^{2}} \log \frac{\tilde{a}-\langle a\rangle-[a](u)}{\tilde{a}-\langle a\rangle}
$$

\section{Appendix III.}

We consider a physical system described by a $N$ component vector field $\omega$, and a partition function $Z(\omega)$ which depends on extra quenched random variables (see Sect. 6). The replica method is supposed to yield as a result :

$$
\overline{Z\left(\omega_{1}\right) . . Z\left(\omega_{n}\right)}=c^{\mathrm{t}} \sum_{\pi} \exp \left(-\frac{1}{2} \sum_{a, b}\left(Q^{-1}\right)_{\pi(a) \pi(b)} \omega_{a} \cdot \omega_{b}\right),
$$

where the $\sum_{\pi}$ runs over all permutations of $n$ elements.

The problem is to deduce the physical distribution of $\omega$,

$$
P(\omega)=\frac{Z(\omega)}{\int \mathrm{d} \omega^{\prime} Z\left(\omega^{\prime}\right)}
$$

and to understand how it fluctuates from sample to sample. The solution is discussed in section 6 , here we just sketch some of the proofs. For simplicity we keep to the case where $Q$ has a single step r.s.b. structure described by the parameters $\tilde{q}, q_{1}, q_{0}$ and $u_{\mathrm{c}}$ (where the notations are similar to those in (5.16)). Let us first consider the average distribution $P(\omega)$, and compute it successively from the replica approach and from the physical distribution described in section 6 . 
With replicas one deduced from (AIII.1) and (AIII.2) :

$$
\begin{aligned}
& \overline{P(\boldsymbol{\omega})}=\lim _{n \rightarrow 0} \int \mathrm{d} \boldsymbol{\omega}_{2} . . \mathrm{d} \boldsymbol{\omega}_{n} \overline{Z(\boldsymbol{\omega}) Z\left(\boldsymbol{\omega}_{2}\right) . . Z\left(\boldsymbol{\omega}_{n}\right)} \\
& =\lim _{n \rightarrow 0} \frac{1}{n} \sum_{c=1}^{n} \int \mathrm{d} \boldsymbol{\omega}_{1} \cdot \mathrm{d} \boldsymbol{\omega}_{n} \delta\left(\boldsymbol{\omega}_{c}-\boldsymbol{\omega}\right) \exp \left(-\frac{1}{2} \sum_{a, b}\left(Q^{-1}\right)_{a b} \boldsymbol{\omega}_{a} \cdot \boldsymbol{\omega}_{b}\right) \\
& =\frac{1}{\left[\sqrt{2 \pi \tilde{q}]^{N}}\right.} \exp \left(-\frac{\boldsymbol{\omega}^{2}}{2 \tilde{q}}\right) .
\end{aligned}
$$

On the other hand the same average distribution can be computed from the physical distribution in (6.5)-(6.9) :

$$
\begin{gathered}
\overline{P(\boldsymbol{\omega})}=\int \mathrm{d} W \mathfrak{T}(\dot{W}) \int D_{q_{0}}\left(\boldsymbol{\omega}_{0}\right) \sum_{\alpha} \dot{W}_{\alpha} \int D_{q_{1}-q_{0}}\left(\boldsymbol{\omega}_{\alpha}\right) \\
\frac{1}{\left[\sqrt{\left.2 \pi\left(\tilde{q}-q_{1}\right)\right]^{N}}\right.} \exp \left(-\frac{\left(\boldsymbol{\omega}-\boldsymbol{\omega}_{\alpha}-\boldsymbol{\omega}_{0}\right)^{2}}{2\left(\tilde{q}-q_{1}\right)}\right)
\end{gathered}
$$

where $D_{q}(\boldsymbol{\omega})$ is a Gaussian measure :

$$
D_{q}(\omega) \equiv \frac{\mathrm{d} \omega}{[\sqrt{2 \pi q}]^{N}} \exp \left(-\frac{\omega^{2}}{2 q}\right) .
$$

Formula (AIII.4) shows that $\omega$ is precisely a Gaussian variable of mean zero and width $\tilde{q}$, as was obtained in (AIII.3) with the replica method.

We now go one step further and show that the results for the average joint distribution of two fields $\boldsymbol{\omega}$ and $\boldsymbol{\omega}^{\prime}$ are identical in the two approaches.

With replicas one must compute :

$$
\begin{array}{r}
\overline{P(\boldsymbol{\omega}) P\left(\boldsymbol{\omega}^{\prime}\right)=} \lim _{n \rightarrow 0} \frac{1}{n(n-1)} \sum_{c \neq c^{\prime}} \int \prod_{a} \mathrm{~d} \boldsymbol{\omega}_{a} \delta\left(\boldsymbol{\omega}_{\mathrm{c}}-\boldsymbol{\omega}\right) \delta\left(\boldsymbol{\omega}_{\mathrm{c}}^{\prime}-\boldsymbol{\omega}^{\prime}\right) \times \\
\quad \exp \left(-\frac{1}{2} \sum_{a, b}\left(Q^{-1}\right)_{a b} \boldsymbol{\omega}_{a} \cdot \boldsymbol{\omega}_{b}\right) .
\end{array}
$$

For a matrix $Q$ with one step r.s.b., there are two types of terms depending on whether $c$ and $c^{\prime}$ are in the same block or in two different blocks. Denoting by $m$ the size of the blocks, one gets :

$$
\begin{aligned}
\overline{P(\boldsymbol{\omega}) P\left(\boldsymbol{\omega}^{\prime}\right)=} & \frac{m}{\sqrt{2 \pi\left(\tilde{q}^{2}-q_{0}^{2}\right)}} \exp \left(-\frac{1}{2}\left(\boldsymbol{\omega}, \boldsymbol{\omega}^{\prime}\right)\left(\begin{array}{ll}
\tilde{q} & q_{0} \\
q_{0} & \tilde{q}
\end{array}\right)^{-1}\left(\begin{array}{l}
\boldsymbol{\omega} \\
\boldsymbol{\omega}^{\prime}
\end{array}\right)\right)+ \\
& +\frac{1-m}{\sqrt{2 \pi\left(\tilde{q}^{2}-q_{1}^{2}\right)}} \exp \left(-\frac{1}{2}\left(\boldsymbol{\omega}, \boldsymbol{\omega}^{\prime}\right)\left(\begin{array}{ll}
\tilde{q} & q_{1} \\
q_{1} & \tilde{q}
\end{array}\right)^{-1}\left(\begin{array}{l}
\boldsymbol{\omega} \\
\boldsymbol{\omega}^{\prime}
\end{array}\right)\right) .
\end{aligned}
$$


The physical distribution derived from (6.5)-(7.9) is :

$$
\begin{aligned}
& \overline{P(\boldsymbol{\omega}) P\left(\boldsymbol{\omega}^{\prime}\right)}=\int \mathrm{d} W \mathfrak{T}(W) \int D_{q_{0}}\left(\boldsymbol{\omega}_{0}\right) \frac{1}{\left[2 \pi\left(\tilde{q}-q_{1}\right)\right]^{N}} \times \\
& \times\left\{\sum_{\alpha \neq \beta} \bar{W}_{\alpha} \dot{W}_{\beta} \int D_{q_{1}-q_{0}}\left(\boldsymbol{\omega}_{\alpha}\right) D_{q_{1}-q_{0}}\left(\boldsymbol{\omega}_{\beta}\right) \exp \left(-\frac{\left(\boldsymbol{\omega}-\boldsymbol{\omega}_{\alpha}-\boldsymbol{\omega}_{0}\right)^{2}}{2\left(\tilde{q}-q_{1}\right)}-\frac{\left(\boldsymbol{\omega}^{\prime}-\boldsymbol{\omega}_{\beta}-\boldsymbol{\omega}_{0}\right)^{2}}{2\left(\tilde{q}-q_{1}\right)}\right)\right. \\
& \left.\quad+\sum_{\alpha} \overline{W_{\alpha}^{2}} \int D_{q_{1}-q_{0}}\left(\boldsymbol{\omega}_{\alpha}\right) \exp \left(-\frac{\left(\boldsymbol{\omega}-\boldsymbol{\omega}_{\alpha}-\boldsymbol{\omega}_{0}\right)^{2}}{2\left(\tilde{q}-q_{1}\right)}-\frac{\left(\boldsymbol{\omega}^{\prime}-\boldsymbol{\omega}_{\alpha}-\boldsymbol{\omega}_{0}\right)^{2}}{2\left(\tilde{q}-q_{1}\right)}\right)\right\}
\end{aligned}
$$

Using the fact $\sum_{\alpha} \overline{W_{\alpha}^{2}}=1-m$, which has been derived in [31], one finds that the two results (AIII.7) and (AIII.8) coincide.

A close look at the equations shows that in the general case of $\overline{P\left(\omega_{1}\right) . . P\left(\omega_{k}\right)}$, the results derived from the two approaches also coincide. This is also true when the breaking of replica symmetry is larger than the one step breaking described here : although the formulae become quite complicated and the results cannot be written in closed from, one can still show that the computations are identical with the two approaches, which establishes the results of section 6 .

\section{References}

[1] Kardar M. and Zhang Y. C., Phys. Rev. Lett. 58 (1987) 2087.

[2] Kardar M., Parisi G. and Zhang Y. C., Phys. Rev. Lett. 56 (1986) 889.

[3] Forster D., Nelson D. R. and Stephen M. J., Phys. Rev. A 16 (1977) 732.

[4] Huse D. A., Henley C. L. and Fisher D. S., Phys. Rev. Lett. 55 (1985) 2924.

[5] Derrida B. and Spohn H., J. Stat. Phys. 51 (1988) 817.

[6] Nattermann T. and Rujan P., Int. J. Mod. Phys. B 3 (1989) 1597 ;

Natterman T. and Villain J., Phase transitions 11 (1988) 5.

[7] Shakhnovich E. I. and Gutin A. M., J. Phys. A 22 (1989) 1647.

[8] Villain J., J. Phys.Lett. France 43 (1982) L551;

Grinstein G. and MA S. K., Phys. Rev. B 28 (1983) 2588 ;

Halpin-Healey T., Phys. Rev. Lett. 62 (1989) 442 ;

Medina E., Hwa T., Kardar M. and Zhang Y. C., Phys. Rev. A 39 (1989) 3053.

[9] EFETOV K. B. and LARKin A., Zh. Eksp. Teer. Fiz. 72 (1977) 2350.

[10] Kogon H. S. and Wallace D. J., J. Phys. A 14 (1981) 527.

[11] BrÉzin E. and ORLAND H. (1986), unpublished.

[12] Parisi G. and Sourlas N., Phys. Rev. Lett. 43 (1979) 744 ;

PARISI G., in Les Houches 1982, Session XXXIX, J. B. Zuber and R. Stora Eds. (North-Holland, Amsterdam, 1984);

PARISI G., in Quantum field theory and quantum statistics. Essays in Honour of the Sixthieth Birthday of E. S. Fradkin, by I. A. Batalin, C. J. Isham and G. A. Vilkovisky Ed., Adam Hilger, Bristol, 1987).

[13] Mezard M., Parisi G. and Virasoro M., Spin glass theory and beyond (World Scientific, Singapore, 1987).

[14] Villain J., J. Phys. Lett. France 43 (1982) 808;

Villain J., Semeria B., Lançon and Billard L., J. Phys. C 16 (1983) 2588 ;

VIllain J. and SEMERIA B., J. Phys. Lett. France 4 (1983) 889 ;

Engel A., J. Phys. Lett. France 46 (1985) 409 ;

Fisher D. S., Phys. Rev. Lett. 56 (1986) 1964 ;

Schulz. U., Villain J., Brézin E. and Orland H., J. Stat. Phys. 51 (1988) 1;

VILlain J., J. Phys. A 21 (1988) L1099. 
[15] Parisi G., J. Phys. France 51 (1990) 1595;

MÉzard M., J. Phys. France 51 (1990) 1831.

[16] Mézard M. and Parisi G., J. Phys. A 23 (1990) L.1229.

[17] De Dominicis C. and Kondor I., Phys. Rev. B 27 (1983) 606.

[18] ZHANG Y. C., Non-universal roughening of kinetic self-affine interfaces (1990) Rome preprint.

[19] Medina E., Kardar M., Shapir Y. and Wang X. R., Phys. Rev. Lett. 62 (1989) 941 ;

ZHANG Y. C., Phys. Rev. Lett. 62 (1989) 979.

[20] Parisi G., R. Acad. Naz. Lincei. XI-1 (1990) 3.

[21] Kardar M., Nucl. Phys. B 290 (1987) 582.

[22] De Almeida J. R. L. and Thouless D. J., J. Phys. A 11 (1978) 983.

[23] MEZARd M. and Parisi G. (1990), in preparation.

[24] Derrida B. and Gardner E., J. Phys. C 19 (1986) 5787 ;

COOK J. and Derrida B., Europhys. Lett. 10 (1989) 195; J. Stat. Phys. 57 (1989) 89.

[25] Parisi G. and Virasoro M. A., J. Phys. France 50 (1989) 3317.

[26] Ruelle D., Comm. Math. Phys. 108 (1987) 225.

[27] FISHER D. S. and HUSE D. A., preprint (1990).

[28] SHAPIR Y., preprint (1990).

[29] Parisi G., J. Phys. A 13 (1980) 1887.

[30] MEZard M. and Parisi G., J. Phys. Lett. France 45 (1985) L707.

[31] Mézard M., Parisi G., Sourlas N., Toulouse G. and Virasoro M., J. Phys. France 45 (1984) 843. 